\title{
Breathing Transitions in MIL-53(Al) Metal-Organic Framework Upon Xenon Adsorption
}

\author{
Anne Boutin, Marie-Anne Springuel-Huet, Andrei Nossov, Antoine Gédéon, Thierry Loiseau, \\ Christophe Volkringer, Gérard Férey, François-Xavier Coudert and Alain H. Fuchs*
}

Porous Metal-Organic Frameworks (MOFs) are a topical class of materials that display an extremely large range of crystal structures and host-guest properties, potentially giving them a major impact in in many areas of science and technology ${ }^{[1 \mathrm{a}-\mathrm{e}]}$. A growing number of these materials show exceptional guest-responsive behaviors upon gas adsorption, due to the flexibility of their hybrid organicinorganic frameworks ${ }^{[1 \mathrm{e}, 2 \mathrm{a}-\mathrm{d}]}$. The MIL-53 materials family ${ }^{[3 \mathrm{a}, \mathrm{b}]}$ has recently attracted a lot of attention on account of its massive flexibility and the occurrence of a double structural transition ("breathing") upon adsorption of some gases $\left(\mathrm{CO}_{2}, \mathrm{H}_{2} \mathrm{O}\right)$ but not others $\left(\mathrm{H}_{2}, \mathrm{CH}_{4}\right)^{[3 \mathrm{c}]}$, at room temperature. It was also reported very recently that these transitions occurred upon gas phase adsorption of several $n$-alkanes ${ }^{[3 \mathrm{~d}]}$ or xylene isomers ${ }^{[3 \mathrm{e}]}$. It has been suggested that apolar species such as methane or rare gases could not induce breathing because of their too low adsorption enthalpies in MIL-53 materials ${ }^{[3 \mathrm{c}]}$. Here, we report a xenon adsorption study in MIL$53(\mathrm{Al})$ in the temperature range $195 \mathrm{~K}-323 \mathrm{~K}$ in which we clearly observe breathing transitions in the measured adsorption isotherms. With the use of a recently developed thermodynamic model, we predict for the first time a phase diagram for xenon adsorption in MIL-53. The present method is very general and could be applied to any other guest-flexible host system.

The MIL-53(Al) framework topology is formed of unidimensional chains of corner-sharing $\mathrm{AlO}_{4}(\mathrm{OH})_{2}$ octahedra linked by 1,4-benzenedicarboxylate (BDC) ligands, which results in linear lozenge-shaped channels large enough to accommodate small guest molecules ${ }^{[3 \mathrm{~b}, \mathrm{f}]}$. The breathing transitions mentioned above are taking place between two forms of this system: a large pore (Ip)

\section{[*] Dr. A. Boutin}

Laboratoire de Chimie Physique

CNRS and Univ. Paris-Sud, 91405 Orsay Cedex (France)

Dr. M.-A. Springuel-Huet, Dr. A. Nossov, Prof. Dr. A. Gédéon Laboratoire Système Interfaciaux à l'Échelle Nanométrique Université Pierre et Marie Curie 75252 Paris Cedex 05 (France)

Dr. T. Loiseau ${ }^{[+]}$, Dr C. Volkringer, Prof. Dr. G. Férey ${ }^{[++]}$ Institut Lavoisier, UMR CNRS 8180 and Université de Versailles St-Quentin en Yvelines, 78035 Versailles (France)

$\left.{ }^{+}\right]$Present address: Unité de Catalyse et de Chimie du Solide, USTL-ENSCL, 59652 Villeneuve d'Ascq (France)

$\left.{ }^{++}\right]$and Institut Universitaire de France, Paris (France)

Dr. F.-X. Coudert, Prof. Dr. A.H. Fuchs

Ecole nationale supérieure de chimie de Paris (Chimie ParisTech), CNRS and Univ. Pierre et Marie Curie 11, rue Pierre et Marie Curie, 75005 Paris (France) E-mail: alain.fuchs@enscp.fr Fax: (+33)1-43292059 structure and a narrow pore (np) one (Figure S1 in the Supporting Information). The MIL-53 materials family has been the subject of numerous structural chemistry studies, but quite less of thorough thermodynamics analysis ${ }^{[1 \mathrm{c}, \mathrm{e}]}$.

In Figure 1, we report experimental xenon adsorption isotherms in MIL-53(Al) at various temperatures. The low-temperature isotherms $(195$ and $220 \mathrm{~K}$ ) display a first step at $\sim 3$ molecules per unit cell and reach a high-pressure plateau at $\sim 8$ to 9 molecules/uc. These values are consistent with the np and the lp structures respectively. The $220 \mathrm{~K}$ isotherm plotted on a log scale is particularly interesting (Figure S2 in the Supporting Information). It demonstrates the existence of two well-defined transitions which, given the large amount of previously published data on MIL-53, can be confidently ascribed to the Ip-np and the np-lp transitions respectively, even though structural data for the $\{\mathrm{Xe}, \mathrm{MIL}-53(\mathrm{Al})\}$ system are not yet available.

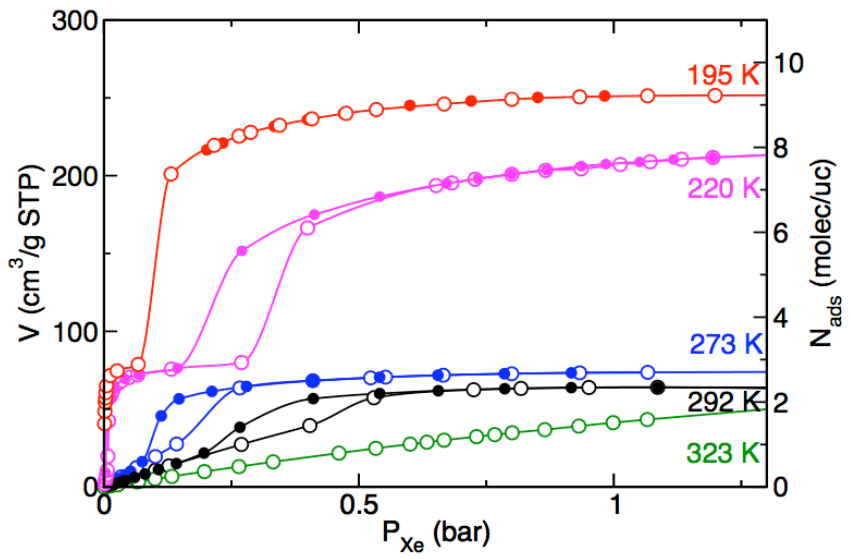

Figure 1. Experimental xenon adsorption isotherms in MIL-53 (AI). Open symbols: adsorption branch; filled symbols: desorption branch. Left scale: measured adsorbed volumes. Right scale: corresponding number of adsorbed molecules per unit cell. The low-pressure desorption branch at $195 \mathrm{~K}$ (hysteresis loop) has not been recorded, for technical reasons.

In the $195 \mathrm{~K}$ adsorption isotherm, the step corresponding to the np-lp transition is clearly visible in Figure 1, but a careful examination of the low pressure regime shows no sign of the lp-np transition. This could either be due to the fact that it occurs at a very low and hardly detectable pressure, or to the fact that the starting empty material is already in the np structure at this temperature. A full thermodynamic analysis will show, further below, that the second guess is the right one.

The 273 and $292 \mathrm{~K}$ isotherms clearly display a phase transition, with a hysteresis loop, which can be attributed to the lp-np transition, given that the high pressure plateaus $(\sim 3$ molec/uc $)$ correspond to the filling of the np structure. The reopening of the 
MIL-53(Al) structure may take place at higher pressure, but was beyond the reach of the present experiments. Finally, the $323 \mathrm{~K}$ isotherm shows no sign of phase transition in the accessible pressure range, and the framework is thus believed to remain in the lp form, since it is known as the most stable state at room temperature and above. We conclude from this first part of the study that, unexpectedly, adsorption of a rare gas can induce structural breathing in MIL-53.

We now turn to the thermodynamic analysis of the data reported in Figure 1. We use the so-called osmotic thermodynamic ensemble which is the appropriate one to describe the adsorption of a fluid in a flexible porous material ${ }^{[4 a]}$. For materials exhibiting clear structural transitions between different metastable framework structures (as opposed to the phenomenon of progressive, continuous swelling for instance), we demonstrated in an earlier work that the use of an "osmotic sub-ensemble" adequately describes the equilibrium between host structures upon fluid adsorption ${ }^{[4 a, b]}$. This model was successfully applied to understand the presence or absence of breathing effects in MIL-53(Al) upon $\mathrm{CO}_{2}, \mathrm{CH}_{4}$ or linear alkanes adsorption at room temperature ${ }^{[4 b, c]}$.

As in our previous studies, we use Langmuir fits of the experimental isotherms as approximations to the "rigid host" isotherms in both $\mathbf{l p}$ and $\mathbf{n p}$ structures. The Langmuir fits are shown in Figure S3 and S4 and the Langmuir parameters used are given in Table S1. We have used the 273 and $292 \mathrm{~K}$ stepped isotherms to determine the transition enthalpy and entropy of the empty host material (see reference $4 \mathrm{~b}$ for calculation method), and found: $\Delta \mathrm{H}_{\text {host }}$ $=\mathrm{H}_{\mathrm{lp}}-\mathrm{H}_{\mathrm{np}}=15 \mathrm{~kJ} \cdot \mathrm{mol}^{-1}$ and $\Delta \mathrm{S}_{\mathrm{host}}=\mathrm{S}_{\mathrm{lp}}-\mathrm{S}_{\mathrm{np}}=74 \mathrm{~J}_{\mathrm{K}} \mathrm{K}^{-1} \cdot \mathrm{mol}^{-1}$. The transition enthalpy value is in fair agreement with the very recent estimation of $\Delta \mathrm{H}_{\text {host }}$ from Devautour-Vinot et al ${ }^{[5 \mathrm{a}]}$ who combined TGA and DSC water desorption analysis of three MIL materials, and found a value of $20 \mathrm{~kJ} \mathrm{~mol}^{-1}$. Provided that the transition enthalpy and entropy can both be considered as constant in the temperature range investigated, one can simply compute the free energy difference between the empty lp and the np structures. Not unexpectedly, the Ip form is predicted to be the most stable one at room temperature. We use the same values to predict that the transition free energy vanishes at $\mathrm{T}=203 \mathrm{~K}$. As pointed out earlier $^{[5 b]}$, one of the interests of the osmotic ensemble model lies in the fact that it enables to compute equilibrium thermodynamic data for the bare host material, using thermodynamic adsorption data only. Here we predict that the np structure becomes the most stable one below $203 \mathrm{~K}$. This is fully consistent with the fact that no lp-np transition was observed in the $195 \mathrm{~K}$ experimental isotherm. At this temperature, the bare material is already in its np form.

Given the approximations of the model and the uncertainties associated with the different data fits, the predicted equilibrium lpnp transition in the empty MIL-53(Al) is obviously subject to a large uncertainty. A crude estimate is $\pm 20 \mathrm{~K}$, something that we will be able to test once adsorption isotherms will be available for other \{guest,MIL-53(Al)\} systems over a wide range of temperatures. Nevertheless, the present prediction is in keeping with the neutron scattering study of the bare MIL-53(Al) performed by Liu et al. ${ }^{[5]}$ who observed a reversible lp-np transition, accompanied by a large hysteresis in the range of 150 to $350 \mathrm{~K}$. The present work thus provides another strong indication that the low-temperature stable form of MIL-53(Al) is the closed np form. There are qualitative (entropic) reasons to believe that the contracted form should be the most stable one at low temperature, but this is at variance with the recent DFT calculations of Coombes et $a l^{[5 \mathrm{c}]}$ who predicted the open Ip form to be the most stable one at zero Kelvin, although the authors noted that the computed potential energy surface was very flat. It should also be mentioned that MIL-53(Ga) and MIL-53(Al) were recently observed in the $\mathbf{n p}$ structure $^{5 \mathrm{~d}}$, upon dehydration at 353 and $333 \mathrm{~K}$ respectively.

We note in passing that the neutron powder diffraction data of Liu et $a l^{[5 b]}$ enable to compute, for the bare material, the "magnitude of breathing" that Llewellyn and coworkers ${ }^{[3 \mathrm{c}]}$ correlated with the van der Waals volume of the guest molecule. This is shown in Figure S5 in the Supporting Information. The limiting value of 39\% breathing for the empty material correlates well with the existing data for different adsorbate systems. We predict, from the value of its van der Waals volume, a magnitude of breathing for xenon of $\sim 23.5 \%$.

In the third part of this study, we use our model together with the fits performed on the experimental xenon adsorption isotherms in order to investigate the full temperature-loading phase diagram of MIL-53(Al). By solving the osmotic thermodynamic equations numerically, we determine for each temperature, whether breathing occurs and if so, what are the transition pressures. For this we need to know how the affinity $\mathrm{K}$ and the maximum of loading $\mathrm{N}_{\max }$ Langmuir parameters change with temperature, for each phase. These are obtained through a simple linear fit of the existing data (Figures S6 and S7 in the Supporting Information). All the parameters needed to compute the $\{\mathrm{Xe}, \mathrm{MIL}-53\}$ phase diagram are given in Table S2 in the Supporting Information.

The predicted temperature-xenon pressure diagram is shown in Figure 2. The lp phase is found to be stable at high temperature and again at lower temperature, with the existence of an intermediate np phase stability domain for xenon pressure lower than a limiting pressure of $\sim 1.6$ bar. This is reminiscent of the re-entrant behavior observed in some liquid crystals ${ }^{[6]}$. As noted above, however, the low-temperature stable phase in absence of xenon (zero pressure) is the np phase.

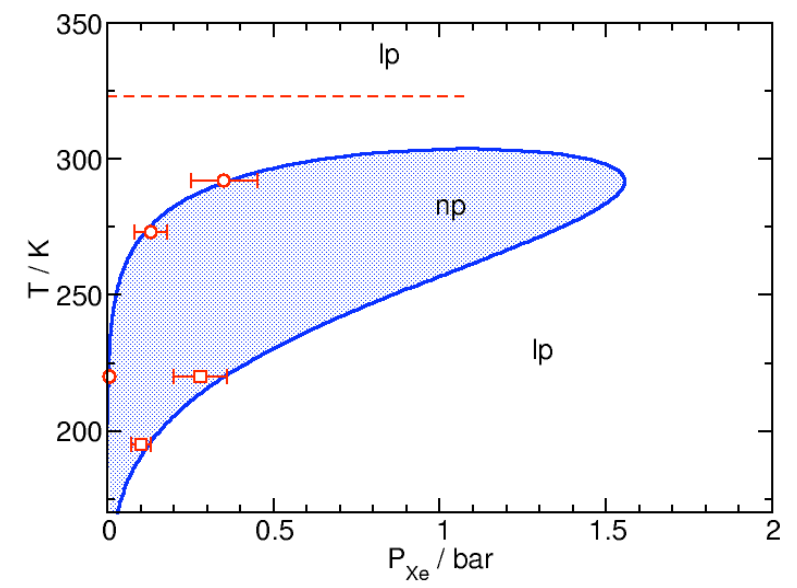

Figure 2. Temperature-xenon pressure phase diagram of MIL-53(Al). Solid line: osmotic thermodynamic model. Open symbols: experimental transition steps in the isotherms. The dashed line corresponds to the experimental temperature $(323 \mathrm{~K})$ for which no transition took place.

Before we turn into the discussion of this diagram, it must be stressed that our model predicts the thermodynamic stability of the phases of a material at full equilibrium and does not take into account hysteresis effects for the time being. Hysteresis was 
systematically encountered in all reported MIL-53 experiments. It often leads to some complicated mixtures of phases effects. In a recent structural study of MIL-53(Fe), Millange et $a l^{7}$ observed intermediate structures which they attributed to a heterogeneous mixtures of crystallites being either in the open or closed form, depending on their contact with the guest molecules. Such a behavior cannot be taken into account in our model, which only describes what would happen in an homogeneous system at complete equilibrium. In Figure S8 of the Supporting Information, we have sketched the same phase diagram with an arbitrary uncertainty of $1 \mathrm{~kJ} \mathrm{~mol}^{-1}$ in the osmotic potential, in order to provide an idea of what this diagram would look like in presence of hysteresis effects. Some theoretical efforts are still needed to capture the mechanistic origin of these large hysteresis effects.

The main features of the phase diagram depicted in Figure 2 can be understood as follows. We start from the equilibrium np-lp temperature at zero pressure of $203 \mathrm{~K}$. The initial slope of the transition curve is positive and rather steep. An examination of the osmotic thermodynamic equations shows that it is proportional to the logarithm of $\left(\mathrm{K}_{\mathrm{np}} / \mathrm{K}_{\mathrm{lp}}\right)$, the ratio of adsorption affinities in the two structures. This term is clearly positive, since the affinity of the guest adsorbate for the closed form of the framework is expected to be higher than for the open form ${ }^{[4 b, c]}$. To put it very simply, an adsorbed species is interacting with both walls in the closed form of MIL-53(Al), while it mainly interacts with one of the walls in the open structure. The $K_{n p} / K_{l p}>1$ condition thus favors the closed $\mathbf{n p}$ phase and consequently the phase transition temperature increases with the xenon loading (i.e. the stability domain of the np phase increases with $\mathrm{P}_{\mathrm{Xe}}$ ). At higher temperature the transition free energy increases and it becomes more and more difficult to maintain the $\mathbf{n p}$ form as the most stable one. This causes the observed bending of the transition line above $250 \mathrm{~K}$. For obvious entropy reasons, the Ip phase will eventually become more stable at high temperature, whatever the xenon loading. This is also true at high pressure. As the adsorbate pressure increases, at any temperature, the Ip structure will eventually become more stable than the $\mathbf{n p}$ one because it can accommodate a higher loading of guest molecules. Since the Ip phase is the most stable one at high enough temperature as well as at high adsorbate pressure, one has to conclude that the stability domain of the np phase should be limited in adsorbate pressure $\left(\mathrm{P}_{\text {lim }} \sim 1.6\right.$ bar in the case of xenon) as seen in Figure 2.

Finally, the loop in the transition line (i.e. the strong decrease in the transition pressure in the low temperature part of the transition line), is due to the fact that the np-lp transition in this domain is taking place at the maximum loading of the np structure. The xenon pressure needed to reach this maximum loading is strongly decreasing at low temperature. Another view of the $\{\mathrm{Xe}, \mathrm{MIL}-$ 53(Al)\} phase diagram is shown in Figure S9 in the Supporting Information. In this figure we have sketched the transition temperatures as a function of loading, i.e. the number of adsorbed xenon atoms in both the np and Ip phase. It shows that the low temperature np-lp transition is indeed taking place at the maximum of loading of the np structure.

The above thermodynamic considerations are very general and are not limited to the special case of xenon adsorption. We expect the main features of the xenon phase diagram to hold for any other \{guest,MIL-53(Al)\} system. The $\mathrm{K}_{\mathbf{n p}} / \mathrm{K}_{\mathbf{l p}}>1$ condition is expected to hold true for all the simple guest molecules that have been investigated so far. This means that there should be a range of temperatures above the equilibrium np-lp transition temperature of the bare MIL-53(Al) material (203 K in our model, subject to the uncertainties described above), where the initially (empty) open structure contracts upon any guest molecule adsorption. The fact that this has not been observed in some cases at room temperature $\left(\mathrm{Ar}, \mathrm{CH}_{4}, \ldots\right)$ might simply mean that this temperature is above the transition line maximum. It is worth mentioning here that the breathing effect in $\{\mathrm{Xe}, \mathrm{MIL}-53(\mathrm{Al})\}$ would not have been detected by a single room temperature adsorption experiment. The present predictions should now be confronted to experimental structural data.

The use of the osmotic thermodynamic model, combined for the first time with a series of gas adsorption experiments at various temperatures, has allowed to shed some new light on the fascinating phase behavior of the MIL-53(Al) flexible material. We derived a generic temperature-loading phase diagram, and we predict that the breathing effect in MIL-53 is a very general phenomenon, that should be observed in a limited temperature range, whatever the guest molecule, since it is expected that the affinity of an adsorbate for the closed np form of the framework will always be higher than for the open Ip structure. We believe that this very general model will provide a useful tool for experimentalists to tune their experimental conditions. Work is in progress to apply this method to the transition behaviors of $\mathrm{CO}_{2}$ and $\mathrm{CH}_{4}$ in MIL-53, as well as to other flexible materials.

\section{Experimental Section}

The MIL-53(Al) sample was prepared as using the synthesis procedure previously described in the literature, following the DMF exchange intermediate route ${ }^{[3]]}$. The xenon adsorption-desorption isotherms were carried out at various temperatures on an automatic ASAP 2020 Micromeretics. Prior to isothermal experiments, the sample (about $150 \mathrm{mg}$ ) was outgassed at $423 \mathrm{~K}$ overnight at a pressure of $10^{-7}$ bar. To obtain the isotherms at different temperatures, the following thermo baths were used: water for $292 \mathrm{~K}$, ice/water for $273 \mathrm{~K}$, dry ice/acetone for $195 \mathrm{~K}$, dry ice/acetonitrile for $220 \mathrm{~K}$. For the isotherm at $323 \mathrm{~K}$, an oven was used. Typically about 30 to 50 data points were measured for each isotherm that took 20 to 40 hours to measure.

Keywords: adsorption · xenon · nanoporous materials $\cdot$ metal-organic frameworks $\cdot$ thermodynamics

[1] a) S. Kitagawa, R. Kitaura, S. Noro, Angew. Chem. Int. Ed. 2004, 43, 2334-2375; b) J.L.C. Rowsell, O.M. Yaghi, Microporous Mesoporous Mater. 2004, 73, 3-14; c) G. Férey, Chem. Soc. Rev. 2008, 37, 191 214; d) J. Long, O.M. Yaghi, Chem. Soc. Rev. 2009, 38, 1213-1214; e) G. Férey, C. Serre, Chem. Soc. Rev. 2009, 38, 1380-1399.

[2] a) S. Kitagawa, K. Uemera, Chem. Soc. Rev. 2005, 34, 109-119; b) A.J. Fletcher, K.M. Thomas, M. Rosseinsky, Solid State Chem. 2005, 178, 2491-2510; c) D. Tanaka, K. Nakagawa, M. Higuchi, S. Horike, Y. Kubota, T.C. Kobayashi, M. Takata, S. Kitagawa, Angew. Chem. Int. Ed. 2008, 47, 3914-3918; d) H.J. Choi, M. Dinca, J. Long, J. Am. Chem. Soc. 2008, 130, 7848-7850.

[3] a) F. Millange, C. Serre, G. Férey, Chem. Comm. 2002, 822; b) T. Loiseau, C. Serre, C. Huguenard, G. Fink, F. Taulelle, M. Henry, T. Bataille, G. Férey, Chem, Eur. J. 2004, 10, 1373-1382; c) P.L. Llewellyn, G. Maurin, T. Devic, S. Loera-Serna, N. Rosenbach, C. Serre, S. Bourelly, P. Horcajada, Y. Filinchuk, G. Férey, J. Am. Chem. Soc. 2008, 130, 12808-12814; d) T.K. Trung, P. Trens, N. Tanchoux, S. Bourelly, P.L. Llewellyn, S. Loera-Serna, C. Serre, T. Loiseau, F. Fajula, G. Férey, J. Am. Chem. Soc. 2008, 130, 16926-16932; e) V. Finsy, C.E.A. Kirschhock, G. Vedts, M. Maes, L. Alaerts, D.E. De 
Vos, G. Baron, J.F.M. Denayer, Chem. Eur. J. 2009, in press; f) C. Serre, S. Bourrely, A. Vimont, N.A. Ramsahye, G. Maurin, P.L. Llewellyn, M. Daturi, Y. Filinchuk, O. Leynaud, P. Barnes, G. Férey, Adv. Mater. 2007, 19, 2246-2251

[4] a) M. Jeffroy, A.H. Fuchs, A. Boutin, Chem. Commun. 2008, 32753277; b) F.-X. Coudert, M. Jeffroy, A.H. Fuchs, A. Boutin, C. MellotDraznieks, J. Am; Chem. Soc. 2008, 130, 14294-14302; c) F.-X.

Coudert, C. Mellot-Draznieks, A.H. Fuchs, A. Boutin, J. Am. Chem. Soc. 2009, 131, 3442-3443.

[5] a) S. Devautour-Vinot, G. Maurin, F. Henn, C. Serre, T. Devic, G. Férey, Chem. Commun. 2009, 2733-2735; b) Y. Liu, J.-H. Her, A.
Dailly, A.J. Ramirez-Cuesta, D.A. Neumann, C.M. Brown, J. Am. Chem. Soc. 2008, 130, 11813-11818; c) D.S. Coombes, F. Cora, C. Mellot-Draznieks, R.G. Bell, J. Phys. Chem. C 2009, 113, 544-552; d) C. Volkringer, T. Loiseau, N. Guillou, G. Férey, E. Elkaïm, A. Vimont, Dalton Trans. 2009, 2241-2249.

[6] P.G. de Gennes, J. Prost, The Physics of Liquid Crystals, Oxford University Press, 2001

[7] F. Millange, C. Serre, N. Guillou, G. Férey, R.I. Walton, Angew. Chem. Int. Ed. 2008, 47, 4100-4105. 